UNIVERSIDADE DE SÃO PAULO

FACULDADE DE EDUCAÇÃO

GLÁUCIA SILVA BIERWAGEN

UMA PROPOSTA DE USO DO BLOG COMO FERRAMENTA DE AUXÍLIO AO ENSINO DE CIÊNCIAS NAS SÉRIES FINAIS DO ENSINO FUNDAMENTAL

São Paulo

2011 
UNIVERSIDADE DE SÃO PAULO

FACULDADE DE EDUCAÇÃO

GLÁUCIA SILVA BIERWAGEN

\section{UMA PROPOSTA DE USO DO BLOG COMO FERRAMENTA DE AUXÍLIO AO ENSINO DE CIÊNCIAS NAS SÉRIES FINAIS DO ENSINO FUNDAMENTAL}

Dissertação submetida ao Programa de Pós-Graduação em Educação da Faculdade de Educação da Universidade de São Paulo como requisito parcial para obtenção de título de Mestre em Educação.

Área de concentração: Ensino de Ciências e Matemática

Orientador: Prof. Dr. Agnaldo Arroio

São Paulo

2011 
AUTORIZO A REPRODUÇÃO E DIVULGAÇÃO TOTAL OU PARCIAL DESTE TRABALHO, POR QUALQUER MEIO CONVENCIONAL OU ELETRÔNICO, PARA FINS DE ESTUDO E PESQUISA, DESDE QUE CITADA A FONTE.

Catalogação na Publicação

Serviço de Biblioteca e Documentação

Faculdade de Educação da Universidade de São Paulo

371.36(81.6 Bierwagen, Glaúcia Silva

B588p Uma proposta de uso do blog como ferramenta de auxílio ao ensino de ciências nas séries finais do ensino fundamental. São Paulo: s.n., 2011. 190 p. Il.

Dissertação (Mestrado) - - Programa de Pós-Graduação em Educação. Área de Concentração: Ensino de Ciências e Matemática - - Faculdade de Educação da Universidade de São Paulo.

1. Tecnologia Educacional 2. Ciência (estudo e ensino) 3. Internet 4. Blog 5.Tecnologia da Informação 6. Escola Pública I Arroio, Aguinaldo, Orient. 


\section{FOLHA DE APROVAÇÃO}

BIERWAGEN, G. S. Uma proposta do uso do blog como ferramenta de auxílio ao ensino de Ciências nas séries finais do Ensino Fundamental. Dissertação apresentada à Faculdade de Educação da Universidade de São Paulo para obtenção do título de Mestre em Educação.

Aprovada em:

\section{Banca Examinadora}

Prof. Dr.

Instituição:

Assinatura:

Prof. Dr.

Instituição:

Assinatura:

Prof. Dr.

Instituição:

Assinatura:

Prof. Dr.

Instituição:

Assinatura:

Prof. Dr.

Instituição: Assinatura: 


\section{Dedicatória}

Dedico, aos meus pais Elion e Dayse, exemplos de muita perseverança e trabalho. São pessoas que me mostraram o caminho para quem eu deveria ser atualmente.

Dedico a Henrique, com muito amor, admiração, gratidão, compreensão, carinho, e presença incansável ao longo período de elaboração deste trabalho. Pela paciência incrível em meus momentos de cansaço e desânimo durante a realização deste projeto.

Dedico a minha querida avó, com meu amor, e apreciação por sua sabedoria, percepção e gentileza que me inspiraram em momentos difíceis, e por isso sou grata. 


\section{Agradecimentos}

Meu sinceros agradecimentos:

A Deus, que conhece meu coração.

A minha família que me apóia e apoiou em todos os momentos pessoais, profissionais e acadêmicos da minha vida.

Ao meu orientador Agnaldo Arroio, aos professores das disciplinas que cursei durante o Mestrado e que me auxiliaram a iniciar o desenvolvimento de conhecimentos sobre tecnologias e em, especial aos professores Marcelo Giordan por trazer na disciplina discussões acerca das teorias socioculturais que muito contribuíram para discutir e analisar os resultados deste trabalho; e a professora Stela Piconéz por trazer em sua disciplina discussões acerca do uso das TIC nas escolas públicas.

Aos meus colegas Maria Helena, Dirceu, Marina, Luciana, Lúcia e a todos os companheiros do curso pela amizade e pelo auxílio em desenvolver trabalhos individuais e em grupos.

Aos meus colegas do grupo de Educadores que permitiram discussões acaloradas sobre questões de formação de professores nas Licenciaturas e da nossa própria formação acadêmica.

A minha colega de trabalho Vânia, professora de Ciências, pelo apoio e incentivo e a todos da escola que também fizeram o mesmo. Pelas colegas Sueli, Sônia, Cleide, Márcia, Luciene e tantas outras da EMEF Prof.a Marili Dias.

A minha colega Evangelina, professora no CEU Anhanguera, pelo apoio nas idéias criaticas e incentivadoras do nosso trabalho como professoras orientadoras de informática educativa.

A nossa formadora de professores orientadores de informática educativa, Renata, pelo apoio e incentivo ao nosso trabalho.

A gestão da EMEF Prof ${ }^{a}$ Marili Dias por não opor-se ao trabalho e apoiar o uso da Sala de Informática.

Aos meus queridos alunos participantes desta pesquisa, tão prestativos, tão gentis, que se empenharam no trabalho do uso do blog. 


\section{Lista de ilustrações}

Figura 1 - Situação de moradia............................................................................. 32

Figura 2 - Gráfico Bens................................................................................ 33

Figura 3 - Gráfico leitura de gêneros textuais........................................................... 34

Figura 4 - Gráfico locais de lazer........................................................................ 34

Figura 5 - Blog "Bioconhecer",........................................................................... 95

Figura 6 - Blog "Ensinando e Vivendo a Vida”........................................................ 96

Figura 7 - Página de inserção do título do blog............................................................ 109

Figura 8 - Página de criação de lista de links e vídeos indicados................................ 110

Figura 9 - Página de criação de links de atividades...................................................... 112

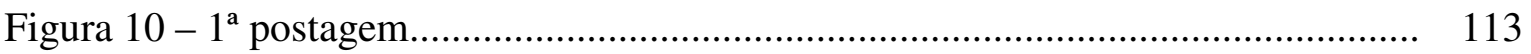

Figura $11-2^{\mathrm{a}}$ postagem............................................................................. 113

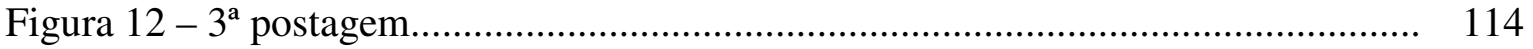

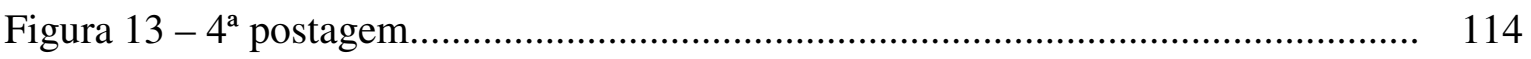

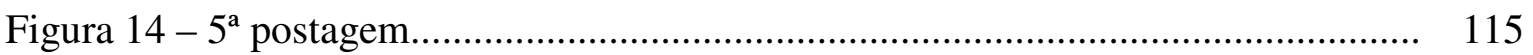

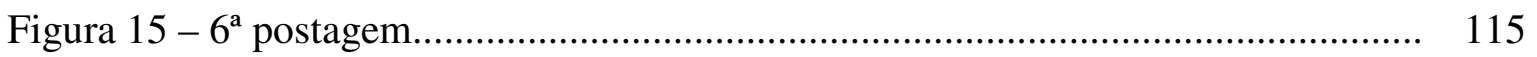

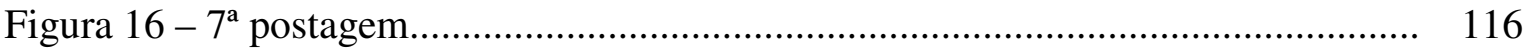

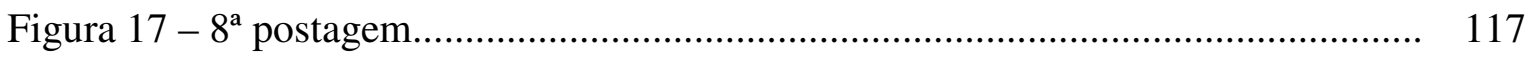

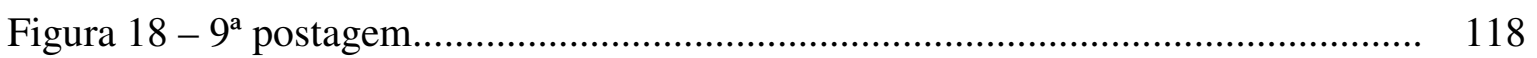

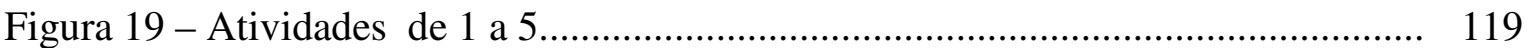

Figura 20 - Atividades de 6 a 10..................................................................... 120

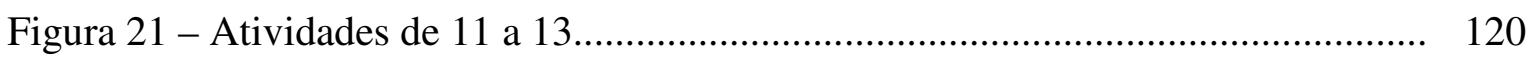

Figura 22 - Atividades 14 e 15..................................................................... 121

Figura 23 - Atividades de 16 a 19.................................................................. 122

Figura 24 - Atividade 20............................................................................... 122

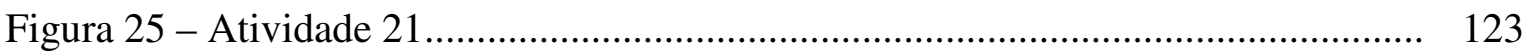

Figura 26 - Atividade 22 ........................................................................... 123

Figura 27 - Atividades 23 a 26...................................................................... 124

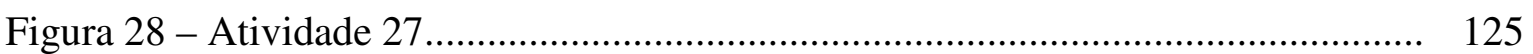

Figura 29 - Diferenças entre sistema reprodutor masculino e feminino...................... 132

Figura 30 - Sistema reprodutor masculino e feminino................................................ 133

Figura 31 - Pesquisa de métodos anticoncepcionais................................................ 138

Figura 32 - Métodos anticoncepcionais.................................................................. 138

Figura 33 - HQ sobre DST........................................................................ 142

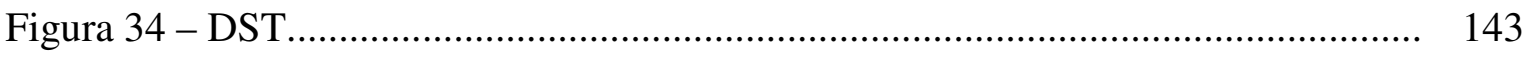

Figura 35 - Relatório do aluno J. ....................................................................... 145

Figura 36 - Relatório do aluno W. ................................................................. 146 


\section{Lista de tabelas}

Tabela 1 - Domínio versus apropriação (WERTSCH, 1998) .................................... 56

Tabela 2 - Relação quanto ao nível de ensino e quantitativo dos resultados

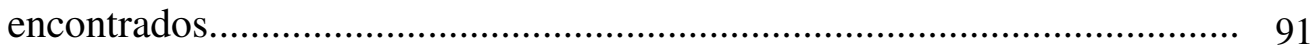

Tabela 3 - Relação de número de blogs quanto as disciplinas no ensino de Ciências.

Tabela 4 - Relação dos blogs e quantitativo dos resultados encontrados quanto aos objetivos de criação

Tabela 5 - Relação dos blogs e quantitativo dos resultados encontrados quanto a utilização pedagógica (GOMES, 2005). 


\section{Lista de siglas}

AIDS - Síndrome da Imunodeficiência Adquirida

AVA - Ambientes Virtuais de Aprendizagem

CIED - Centro de Informática Educativa

DST - Doenças Sexualmente Transmissíveis

EDUCOM - Educação com Computadores

EMEF - Escola Municipal de Ensino Fundamental

EUA - Estados Unidos da América

HQ - História em Quadrinhos

HPV - Papilona Vírus Humano

KM - Quilômetro

LEIA - Laboratório de Educação e Informática Aplicada

MEC - Ministério da Educação e Cultura

NTE - Núcles de Tecnologias Educacionais

PCN - Parâmetros curriculares Nacionais

PROINFO - Programa Nacional de Informática Educativa na Educação

TIC - Tecnologias de Informação e Comunicação

UAB - Universidade Aberta do Brasil

UFMG - Universidade Federal de Minas Gerais

UFPE - Universidade Federal de Pernambuco

UFRGS - Universidade Federal do Rio Grande do Sul

UFRJ - Universidade Federal do Rio de Janeiro

UFSCar - Universidade Federal de São Carlos

UNESCO - Organização das Nações Unidas para a Educação, a Ciência e a

Cultura

UNICAMP - Universidade Estadual de Campinas

UNIFESP - Universidade Federal de São Paulo

WMV - Windows Movie Maker

ZDP - Zona de Desenvolvimento Proximal 


\section{Lista de anexos}

Anexo A - Tabela da relação dos endereços eletrônicos dos blogs pesquisados de acordo com a disciplina.

Anexo B - Solicitação de informação sobre número de blogs cadastrados no Blog blogs

Anexo C - Página do blog da EMEF Prof.a Marili Dias - Parte a.................................... 164

Anexo D - Página do blog da EMEF Prof ${ }^{a}$ Marili Dias - Parte b...................................... 164

Anexo E - Página do blog da EMEF Prof ${ }^{a}$ Marili Dias - Parte c..................................... 165

Anexo F - Página de criação de um nome de um blog.................................................... 165

Anexo G - Página de escolha do modelo de um blog..................................................... 166

Anexo H - Página de personalização de um blog............................................................ 166

Anexo I - Página que identifica a criação do blog.......................................................... 167

Anexo J - Página da aba de edição de postagens............................................................ 167

Anexo K - Página da aba de edição de comentários......................................................... 168

Anexo L - Página da aba de configurações do blog.......................................................... 168

Anexo M - Página da aba gerar receita.......................................................................... 169

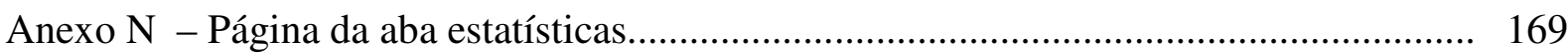

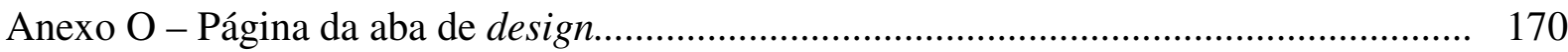

Anexo P - Página da aba design com destaque para a aba visualizar blog........................ 170

Anexo Q - Página Adicionar um gadget - Parte a.......................................................... 171

Anexo R - Página Adicionar um gadget - Parte b....................................................... 171

Anexo S - Link da página sobre aparelho reprodutor masculino.................................... 172

Anexo T - Link da página do vídeo sobre aparelho masculino.......................................... 172

Anexo U - Link da página sobre sistema reprodutor feminino....................................... 173

Anexo V - Link da página de vídeo sobre sistema reprodutor feminino.......................... 173

Anexo W - Link da página de vídeo de humor sobre a fecundação................................... 174

Anexo Y - Link da página sobre ciclo menstrual........................................................... 174

Anexo X - Link da página do vídeo sobre desenvolvimento do feto............................... 175

Anexo $\mathrm{Z}$ - Link da página de informações sobre gravidez durante a gestação e parto....... 175

Anexo AA - Link da página sobre gravidez na adolescência.......................................... 176

Anexo BB - Link da página gravidez semana a semana................................................. 176

Anexo CC - Link da página de vídeo sobre parto normal............................................. 177

Anexo DD - Link da página sobre métodos anticoncepcionais....................................... 177

Anexo EE - Link da página sobre doenças sexualmente transmissíveis............................ 178

Anexo FF - Link da página de História em Quadrinhos (HQ) sobre doenças

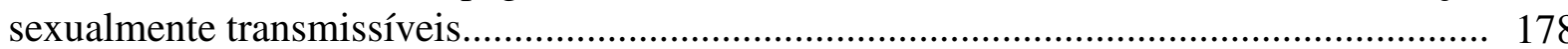

Anexo GG - Link da página do tutorial sobre sexualidade humana................................ 179

Anexo HH - Link da página de modelo de relatório científico.......................................... 179 
Anexo II - Link da página de modelo de relatório.

Anexo JJ - Criação de conta Google.

Anexo KK - Indicação de planilha de apresentação em branco.

Anexo LL - Criação em editor de texto do Google Docs..

Anexo MM - Publicação de atividades na web.

Anexo NN - Criação de endereço eletrônico para ser publicado na web. 182

Anexo OO - Criação de slide em branco.

Anexo PP - Publicação de slide.

Anexo QQ - Criação de endereço eletrônico de slide para ser publicado na web. 184

Anexo RR - Gráfico tipo de transporte de alunos da EMEF Prof ${ }^{a}$ Marili Dias. 184

Anexo SS - Pesquisa sobre DST. 185

Anexo TT - Dados de pesquisa de DST. 185

Anexo UU- Pesquisa de opinião dos alunos - Parte a. 186

Anexo VV - Pesquisa de opinião dos alunos - Parte b. 186

Anexo WW - Pesquisa de opinião dos alunos - Parte c 187

Anexo YY - Planilha de respostas dos alunos 187

Anexo XX - Fotos da Sala de Informática da EMEF Prof ${ }^{\text {a }}$ Marili Dias. 188

Anexo ZZ - Fotos do entorno e do interior da EMEF Prof ${ }^{a}$ Marili Dias. 189

Anexo AAA - Modelo de autorização para participação de alunos em pesquisa acadêmica. 


\section{Sumário}

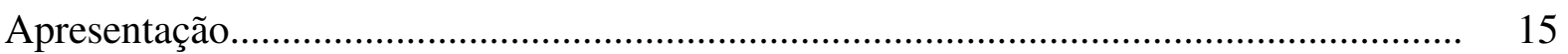

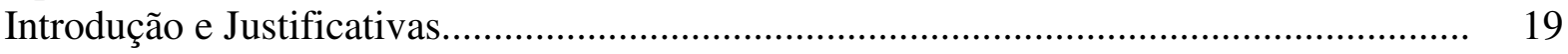

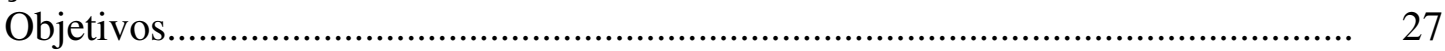

Capítulo 1 - O objeto da pesquisa e a escolha do campo................................................ 29

1.1 - Base filosófica e tipo de pesquisa................................................................. 29

1.2 - Caracterização do local e sujeitos da pesquisa............................................. 31

1.3 - Instrumentos de geração de dados................................................................ 35

Capítulo 2 - Revisão teórica...................................................................................... 37

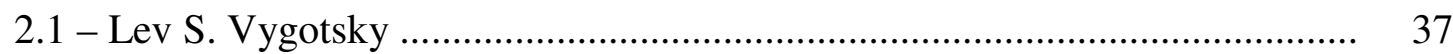

2.2 - Mikhail Bakhtin.................................................................................... 46

2.3 - James Wertsch...................................................................................... 52

2.4 - Concepção de trabalho por projetos............................................................... 56

2.5 - Metodologia WebQuest......................................................................... 61

Capítulo 3 - Tecnologia da informação e comunicação (TIC) e ensino de Ciências......... 66

3.1 - Concepção de Ciências, Técnica e Tecnologia - algumas considerações..... 66

3.2 - Tecnologia da informática no cenário atual e legislação educacional........... 69

3.3 - O ensino de Ciências no Brasil atualmente................................................... 74

3.4 - Histórico e conceituação de tecnologias de informação e comunicação (TIC) na educação.................................................................................. 76

Capítulo 4 - Blogs

4.1 Histórico, conceituação e potencial pedagógico dos blogs............................... 82

4.2 - O blog como ferramenta pedagógica............................................................ 87

4.3 - O blog como ferramenta pedagógica no ensino de Ciências......................... 90

Capítulo 5-Blogs associados aos usos de outras ferramentas tecnológicas e pedagógicas......................................................................................... 97

5.1 - Windows Movie Maker e Youtube .................................................... 97



5.3 - História em quadrinhos (HQ)................................................................. 102

Capítulo 6 - Procedimentos de elaboração do blog e uso do mesmo pelos alunos............ 106

6.1 - Elaboração do blog................................................................................ 106

6.2 - O uso do blog pelos alunos.................................................................. 118

Capítulo 7 - Resultados e discussão............................................................................ 126

Capítulo 8 - Considerações finais............................................................................... 147

Referências bibliográficas................................................................................... 154

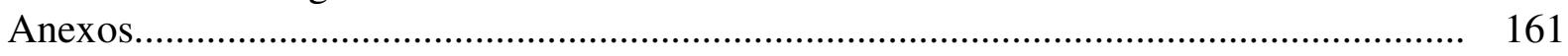


BIERWAGEN, Gláucia Silva. Uma proposta do uso do blog como ferramenta de auxílio ao ensino de Ciências nas séries finais do Ensino Fundamental. 2011. 190 f. (Dissertação de Mestrado) - Faculdade de Educação, Universidade de São Paulo, São Paulo, Brasil, 2011.

\section{RESUMO}

Esta dissertação tem por objetivo explicitar como o trabalho com o blog, utilizando outras ferramentas tecnológicas como o e-mail, Google Docs, HQ digital online podem auxiliar aos alunos produzirem atividades na disciplina de Ciências do Ensino Fundamental do ciclo II, com a temática da sexualidade. As atividades buscaram estimular habilidades como reflexão, autonomia, pesquisa, raciocínio lógico, trabalhar de modo cooperativo e ler e escrever utilizando diferentes gêneros textuais. Busca ainda contemplar a participação ativa destes alunos na construção do seu aprendizado por meio das ferramentas tecnológicas citadas anteriormente. Para o desenvolvimento do trabalho buscou-se abordar fundamentos teóricos socioculturais como os dos pesquisadores Vygostky (formação de conceitos espontâneos e científicos, zona de desenvolvimento proximal, funções psicológicas superiores e mediação simbólica), Bakhtin (enunciação, gêneros discursivos e polifonia) e Wertsch (teoria da ação mediada, ferramentas culturais, domínio e apropriação de conhecimentos). Para a elaboração da estrutura física do blog buscou-se apoiar na concepção de trabalho por projetos (desenvolvendo-se o tema da sexualidade) e na metodologia WebQuest (utilizando-se alguns elmentos da mesma como: pesquisa na internet, desenvolvimento de tarefas, divulgação das atividades realizadas pelos alunos, passo a passo de atividades). Conceitua e aborda historicamente as TIC e blogs na educação e no ensino de Ciências. Relaciona as TIC com o ensino de Ciências e a legislação educacional. Apresenta outras ferramentas tecnológicas que podem ser associadas ao uso do blog como: Windows Movie Maker, Youtube, Gmail, Google Docs, HQ digital online. O presente trabalho mostra como foi todo o processo de desenvolvimento de um blog (com o nome "Aprender Ciências - Sexualidade") feito por uma professora da rede pública em uma escola na cidade de São Paulo, mostrando como a mesma aplicou o uso deste blog com um grupo de alunos de uma $7^{\text {a }}$ série $\left(8^{\circ}\right.$ ano, no atual ensino de 9 anos) e quais foram as principais atividades desenvolvidas por estes. A elaboração das orientações das atividades feitas pela professora para os alunos pode ser visualizada por meio de postagens e links no blog e as atividades realizadas pelos alunos por meio das postagens e links contidos nestas, também no blog. Esta pesquisa busca relacionar, analisar e discutir a elaboração do blog produzido pela professora e o uso do blog realizado por este grupo de alunos a partir dos pressupostos teóricos apontados anteriormente: teorias socioculturais, concepção de trabalho por projetos e metodologia WebQuest.

Palavras-chaves: blog, teorias socioculturais, tecnologias da informação e comunicação 
BIERWAGEN, Gláucia Silva. A proposal to use the blog as a tool to aid the teaching of science in the final grades of elementary school. 2011. 190 f. (Master's Dissertation) Faculty of Education, University of São Paulo, São Paulo, Brazil, 2011.

\begin{abstract}
This dissertation aims to explain how the work with the blog, using other technology tools such as email, Google Docs, Digital HQ can help students produce activities in the discipline of Natural Science at Elementary School II with the theme of sexuality. The activities sought to encourage skills such as reflection, autonomy, research, logical reasoning, working cooperatively and to read and write using different text genres. Search still see the active participation of students in shaping their learning these technological tools mentioned above. For the development of the work sought to address theoretical foundations of sociocultural researchers as Vygotsky (formation of spontaneous and scientific concepts, zone of proximal development, higher psychological functions and symbolic mediation), Bakhtin (enunciation, genres and polyphony) and Wertsch (theory of action mediated, cultural tools, mastery and appropriation of knowledge). In developing the physical structure of the blog was sought to support the design work for projects (developing the theme of sexuality) and methodology WebQuest (using some elements the same as Web search, job development, dissemination activities performed by the students, step by step activities). Conceptualizes and discusses historically and blogs on ICT in education and science teaching. ICT relates to the teaching of science and education legislation. There are other technological tools that can be associated with the use of the blog as Windows Movie Maker, Youtube, Gmail, Google Docs and Digital HQ. This paper shows how the whole process of developing a blog (with the name "Learning Sciences - Sexuality") made by a teacher in a public school in São Paulo city, showing how it applied the use of this blog with a group of students from a 7th grade (thirteen and fourteen old) and what were the main activities developed by them. The preparation of guidelines for activities done by the teacher for students can be viewed through the blog posts and links, and activities undertaken by students through the posts and links contained in these, also on the blog. This research seeks to relate, analyze and discuss the preparation of the blog produced by the teacher and the use of the blog done by this group of students from the theoretical assumptions outlined earlier, sociocultural theories, design work for projects and methodology WebQuest.
\end{abstract}

Key-words: blog; sociocultural theories; information and communication technologies 